\title{
CIRSE Position Statement: Interventional Radiologists and Intra- arterial Stroke Therapy
}

\author{
Hans van Overhagen ${ }^{1}$ - Wim H. van $\mathrm{Zwam}^{2} \cdot$ Antonin Krajina $^{3} \cdot \mathrm{Jens}_{\text {Fiehler }}{ }^{4}$. \\ Jim A. Reekers ${ }^{5} \cdot$ Saruhan Cekirge $^{6} \cdot$ John Thornton $^{7} \cdot$ Christoph A. Binkert $^{8}$. \\ Elias Brountzos 9 - Afshin Gangi ${ }^{10} \cdot$ Robert Morgan ${ }^{11}$
}

Published online: 7 August 2018

(C) Springer Science+Business Media, LLC, part of Springer Nature and the Cardiovascular and Interventional Radiological Society of Europe (CIRSE) 2018

Recent trials have shown the clinical benefit of endovascular thrombectomy (EVT) in patients who have suffered from an acute ischaemic stroke due to large vessel occlusion of the anterior intracranial circulation [1-8]. Successful intra-arterial stroke therapy reduces the number of patients who will be dependent on care or live in nursing homes. This will lead to significant cost savings in social

Robert Morgan

Robert.Morgan@stgeorges.nhs.uk

Hans van Overhagen

hansvo@xs4all.nl

Wim H. van Zwam

w.van.zwam@mumc.nl

Antonin Krajina

antonin.krajina@fnhk.cz

Jens Fiehler

fiehler@uke.de

Jim A. Reekers

j.a.reekers@amc.uva.nl

Saruhan Cekirge

scekirge@gmail.com

John Thornton

johnthornton@beaumont.ie

Christoph A. Binkert

christoph.binkert@ksw.ch

Elias Brountzos

ebrountz@med.uoa.gr

Afshin Gangi

gangi@unistra.fr

1 Haga Teaching Hospital, Den Haag, The Netherlands care budgets across Europe, rendering the treatment highly cost-effective [9].

As a result of these evidence-based favourable outcomes, there is an increasing demand to adapt acute stroke management systems in order to make EVT available for as many acute ischaemic stroke patients as possible. Physicians with experience in neurointerventions perform EVT, as they are not only familiar with neurovascular

2 Maastricht University Medical Center, Maastricht, The Netherlands

3 Charles University Hospital, Hradec Kralove, Czech Republic

4 University Medical Center Hamburg-Eppendorf, Hamburg, Germany

5 Academic Medical Center Amsterdam, Amsterdam, The Netherlands

6 Interventional Neuroradiology, Yuksek Ihtisas University Koru Private Hospitals and Bayindir Private Hospitals, Ankara, Turkey

7 Beaumont Hospital, Dublin, Ireland

8 Kantonsspital Winterthur Medizinische Poliklinik, Winterthur, Switzerland

9 School of Medicine, National and Kapodistrian University of Athens, Athens, Greece

10 University Hospital of Strasbourg, Strasbourg, France

11 St George's University Hospitals NHS Foundation Trust, London, UK 
pathologies and anatomy but also with the tools used in neurointerventions. However, the number of trained neurointerventionists is too low in most countries to cope with the required rapid increase in EVT procedures. An estimated 50-80 non-ischaemic stroke neurointerventional procedures per million people are currently performed annually in the Western world. It is estimated that the amount of people who will require EVT will increase to 120-150 patients per million per year. Clearly, this will have serious implications for the ability of the available trained neurointerventionists to deliver this therapy to these increased numbers.

To meet this growing demand for EVT, a pragmatic approach should be taken in which physicians who already possess some of the required skills can be trained to perform EVT without compromising on quality or patient safety.

Interventional radiologists (IRs) are especially suited to fulfil this role. With their background, knowledge and catheter skills, IRs are very likely to have a considerably shorter learning curve in EVT procedures than other medical specialists. Interventional radiologists who already perform vascular interventions have extensive knowledge and experience in arterial access, small and large vessel catheterisation and recanalisation, thrombectomy, and embolisation. In addition, they are familiar with all of the tools used for these procedures, including vascular sheaths, catheters, microcatheters, guiding systems, guidewires, microguidewires, thrombolytic medication, thrombectomy devices and closure devices. Because of their training as radiologists, they are familiar with neuroanatomy, CT/CTA and MRI/MRA images of brain and the intracranial and extracranial arterial and venous systems of the head and neck.

Interventional radiologists are present in all European countries and in most regions of the Western world. Most IRs are willing and eager to learn new treatment techniques. In many countries, IRs are already performing EVT and are a valuable part of stroke management systems in hospitals where EVT is implemented. Hitherto, because no established training guidelines were available, IRs and neurointerventionists have arranged their own training requirements according to their specialities and local situations. The European neuroradiologists (ESNR) and neurointerventionists (ESMINT) have recently produced their own guidelines, which were recently discussed within the UEMS. The UEMS discussions concluded that in regions where no $24 / 7$ neurointerventional EVT is available, IRs should be able to perform endovascular thrombectomy in patients with acute ischaemic stroke.
Until international quality assurance guidelines become available, principles already established by local quality guidelines on EVT should be adhered to. These principles are:

1. In order to perform endovascular thrombectomy, interventional radiologists should have clear knowledge and understanding of the pathophysiology of stroke, the principles of stroke management, the appearances of stroke on diagnostic imaging (CT, MR, Angiography), and more specifically interpretation of the imaging modalities relevant to EVT. Interventional radiologists should also have knowledge and practical experience of the techniques for carrying out EVT stroke treatment and aftercare.

2. Training of Interventional radiologists for EVT stroke treatment should therefore be based on the following pillars:

- Clinical training in stroke neurology

- Training in neuroimaging, CT(A and P), MR(A) and Angiography

- Training in endovascular stroke treatment

3. A database about procedures, complications and outcomes should be kept. These outcomes should be compared with the outcomes of other units performing EVT on an annual basis.

\section{Conclusions}

In summary, CIRSE believes that interventional radiologists who regularly perform vascular procedures in the peripheral vascular system are uniquely placed to help with the increased demand for intra-arterial stroke therapy prompted by the evidence in favour of EVT from recent landmark randomised controlled trials.

Interventional radiologists are already performing EVT in many centres in Europe, and their continued involvement in EVT is to be encouraged.

Interventional radiologists with the appropriate endovascular skillset and knowledge, who want to learn to perform EVT, should be allowed to do this by consultation and additional training with their local neurointerventional and interventional colleagues.

CIRSE will continue to endeavour to collaborate with our neurointerventional colleagues to produce practical joint intersociety guidelines to define criteria for practitioners of intra-arterial stroke therapy, allowing for their specific knowledge and experience. 
Funding This study was not supported by any funding.

\section{Compliance with Ethical Standards}

Conflict of interest R Morgan currently holds the position of President at CIRSE. Furthermore, C. Binkert, E. Brountzos and A. Gangi are members of CIRSE's Executive Board. Hans van Overhagen and Wim H. van Zwam co-chair CIRSE's Stroke Task Force. J. Fiehler is an Executive Board member of ESMINT and DGNR, a consultant for Acandis, Boehringer Ingelheim, Cerenovus, Covidien, Medtronic, Microvention, Penumbra, Route92, Stryker, and part of the Research Support for EU, BMBF, Medtronic, Microvention, Route92, Stryker. Other authors declare that they have no conflict of interest.

Consent for Publication For this type of study consent for publication is not required.

Ethical Approval For this type of study formal consent is not required.

Informed Consent For this type of study informed consent is not required.

\section{References}

1. Berkhemer OA, et al. A randomized trial of intraarterial treatment for acute ischemic stroke. N Engl J Med. 2015;372(1):11-20.
2. Bracard S, et al. Mechanical thrombectomy after intravenous alteplase versus alteplase alone after stroke (THRACE): a randomised controlled trial. Lancet Neurol. 2016;15(11):1138-47.

3. Campbell BC, et al. Endovascular therapy for ischemic stroke with perfusion-imaging selection. N Engl J Med. 2015;372(11): 1009-18.

4. Goyal M, et al. Randomized assessment of rapid endovascular treatment of ischemic stroke. N Engl J Med. 2015;372(11): 1019-30.

5. Goyal M, et al. Endovascular thrombectomy after large-vessel ischaemic stroke: a meta-analysis of individual patient data from five randomised trials. Lancet. 2016;387:1723-31.

6. Jovin TG, et al. Thrombectomy within 8 hours after symptom onset in ischemic stroke. N Engl J Med. 2015;372(24):2296-306.

7. Muir KW, et al. Endovascular therapy for acute ischaemic stroke: the Pragmatic Ischaemic Stroke Thrombectomy Evaluation (PISTE) randomised, controlled trial. J Neurol Neurosurg Psychiatry. 2017;88(1):38-44.

8. Saver JL, et al. Stent-retriever thrombectomy after intravenous t-PA vs. t-PA alone in stroke. N Engl J Med. 2015;372(24): 2285-95.

9. Sevick LK, et al. Systematic review of the cost and costeffectiveness of rapid endovascular therapy for acute ischemic stroke. Stroke. 2017;48(9):2519-26. 